\title{
Adquisición de señales SEMG con electrodos secos para el control de movimiento de dedos en una prótesis robótica fabricada en una impresora 3D
}

\author{
Acquisition of SEMG signals with dry electrodes for the control of \\ fingers movement in a robotic prosthetics made in a $3 D$ printer \\ Victor Alvarado Castillo ${ }^{1} \quad$ Josué Sánchez Flores ${ }^{1} \quad$ Jhon Carlos Gómez ${ }^{1}$ \\ Efraín Chihuan Huayta ${ }^{1}$ Celso De La Cruz Casaño ${ }^{2 *}$ \\ Recibido 03 de mayo de 2018, aceptado 20 de agosto de 2018. \\ Received: May 03, 2018, Accepted: August 20, 2018
}

\begin{abstract}
RESUMEN
Este artículo presenta la comparación de dos tipos de electrodos, húmedos del tipo $\mathrm{Ag} / \mathrm{AgCl}$ y secos de acero Inoxidable, y el desarrollo de la adquisición y filtrado de señales SEMG (Señales electromiográficas superficiales) para el control de una prótesis robótica, fabricada en una impresora 3D, para personas con amputación del miembro superior. El trabajo muestra que es factible la utilización de electrodos secos de acero inoxidable para la correcta obtención de las señales del músculo y el control de la prótesis. Además, se muestra la posibilidad de realizar el movimiento de dedos en forma secuencial mediante el uso de un solo sensor (conformado por tres electrodos en un solo módulo y un amplificador de señales) utilizando un filtro pasa bajo digital. Los resultados de esta investigación servirán para el desarrollo de prótesis más avanzadas y con componentes comunes para personas con discapacidad, y así, estas personas puedan tener una mejor calidad de vida.
\end{abstract}

Palabras clave: Electromiografía, prótesis de mano, señales SEMG, control de dedos.

\begin{abstract}
This paper presents the comparison of two types of electrodes, wet of type $\mathrm{Ag} / \mathrm{AgCl}$ and dry of stainless steel, and the development of the acquisition and filtering of SEMG signals (surface Electromyography signals) for the control of robotic prosthetic printed in $3 D$ for people with amputation of the upper limb. The work shows that the use of dry electrodes of stainless steel for the correct acquisition of muscle signals and the control of the prosthetic is viable. In addition, the possibility of performing the sequential movement of fingers using a single sensor (made up with three electrodes in only one module and a signal amplifier) using a low-pass filter is also shown. The results of this research will be useful for the development of more advanced and lower cost prosthetics for people with disabilities, and thus, these people can have a better quality of life in the future.
\end{abstract}

Keywords: Electromyography, hand prosthetics, SEMG signals, finger control.

\footnotetext{
1 Carrera de Ingeniería Mecatrónica. Universidad Continental.E-mail: 46927485@continental.edu.pe; 76410112@continental.edu.pe; 75278434@continental.edu.pe; 77340369@continental.edu.pe

2 Unidad de Investigación de la Facultad de Ingeniería. Universidad Continental. E-mail: celsodelacruz@gmail.com

* Autor de correspondencia: celsodelacruz@gmail.com
} 


\section{INTRODUCCIÓN}

En los últimos años, se han visto numerosos avances en lo referente a prótesis robóticas altamente similares en apariencia y con prestaciones cada vez más cercanas a las de una extremidad real [1], [2-3]. Actualmente, en varios lugares del mundo, se realizan investigaciones que proporcionan gran cantidad de desarrollos con el fin de mejorar las prótesis de extremidad superior, mediante el uso de materiales inteligentes, sensores, actuadores y aplicaciones para la adquisición, filtrado y manejo de señales mioeléctricas residuales, normalmente de los músculos del muñón, para el control efectivo de una prótesis de mano [4-6].

Entre los avances realizados recientemente, podemos encontrar el realizado en Japón por la compañía Exii [7], quienes diseñaron un prototipo de un valor aproximado de 30.000 yenes (220 euros). Esta prótesis permite coger objetos con facilidad sin emplear complejos y costosos sistemas, como la conexión cerebral o el movimiento independiente de cada dedo mecánico. Esta simplicidad técnica, permite construir modelos más económicos.

Otro de los trabajos realizados recientemente se tituló "Mano controlada por señales musculares" y fue presentado en Uruguay en septiembre de 2015 [8]. En esta investigación se desarrolló un prototipo de prótesis donde se logró adquirir y visualizar las señales electromiográficas superficiales, en adelante señales SEMG, mediante electrodos y después se procesó y optimizó. Como resultado la amplitud de la señal estaba en el rango de los parámetros para su conversión a señales digitales. Se logró un procedimiento para determinar la relación entre fuerza e intensidad muscular e implementó el código para control de un motor, permitiendo el movimiento continuo de la mano.

Las prótesis de mano también se pueden controlar mediante señales EEG (electroencefalografía) como en la investigación [2]. En ese artículo se muestra el uso de un gorro EEG, el software LabView, el Arduino uno y una prótesis de mano robótica. Sin embargo, utiliza una computadora (PC) para realizar el procesamiento de las señales, lo cual es una desventaja para prótesis reales por el tamaño de la PC. Esta desventaja también lo presenta el trabajo [1], el cual utiliza un módulo de adquisición de las señales SEMG, una PC con software Lab Windows CVI y una mano robótica.

El trabajo de Quinayás [3]. presenta un sistema embebido que detecta en tiempo real la intención de movimiento para el control de una prótesis de mano robótica. Los resultados muestran que el sistema puede ser efectivo para el control de cuatro primitivas motoras.

Para adquirir las señales musculares se tienen dos tipos de electrodos, electrodos secos y electrodos húmedos [9-11]. Los electrodos húmedos permiten una mejor adquisición de la señal, debido a que la impedancia entre el electrodo y la superficie de la piel es constante, sin embargo, tiene la desventaja de la necesidad de humedecer constantemente con un gel especial. Los electrodos secos no necesitan ser humedecidos y no causan malestar en el usuario, sin embargo, existen electrodos secos que no tienen constante la impedancia. La impedancia en los electrodos secos es alta al inicio y a medida que pasan los minutos va disminuyendo; luego de aproximadamente 15 minutos esta impedancia es casi constante [11].

A diferencia de los trabajos anteriores, la presente investigación se centró en el desarrollo de electrodos secos de acero inoxidable, realizar un control de los dedos de la prótesis mediante el uso de un solo sensor y la comparación frente al electrodo húmedo. El sensor está conformado por tres electrodos secos dispuestos en un solo módulo y un amplificador de señales. El objetivo fue demostrar que con componentes comunes se puede obtener un buen desempeño del control de los dedos de la prótesis y, además, demostrar que la disminución de la impedancia del electrodo seco no perjudica el control de la prótesis, por el contrario, mejora el control a medida que baja la impedancia.

Se espera que este nuevo sistema pueda ser utilizado para prótesis comerciales para que más personas puedan acceder a esta tecnología por ser de bajo costo y mejorar su calidad de vida. Además, se espera que esta investigación pueda servir de base para futuras investigaciones y se logre el desarrollo de prótesis $100 \%$ funcionales y económicas. 


\section{PRÓTESIS ROBÓTICA}

\section{Materiales}

- Prótesis impresa con tecnología 3D: constituidas por 30 partes.

- Servomotor SG-5010 6kg: utilizado para replicar el movimiento de los tensores de los dedos.

- Arduino nano: microcontrolador encargado de convertir la señal analógica a digital y generar la señal PWM que será enviada a los servomotores.

- Amplificador de señales SEMG para Arduino "Muscle sensor v3.0": amplifica y filtra los impulsos eléctricos producidos al realizar la contracción muscular.

- Electrodos/placa metálica: conductor eléctrico utilizado para hacer contacto con la superficie externa del músculo.

\section{Sistema mecánico}

El diseño final de la prótesis es la suma de la mano del robot INMOOV [12] y la modificación del sistema de accionamiento de los dedos para que se adapte a personas con un nivel de amputación a través del tercio proximal (ver Figura 1). Con esto, podemos tener un control independiente para cada dedo. El diseño final fue impreso en 3D y en su totalidad en el laboratorio FabLab de la Universidad Continental [13], tomando un tiempo estimado de 42:17 horas y utilizando 467.29 gramos de material. En la Figura 2, se puede observar la prótesis terminada.

\section{Nuevo sensor desarrollado}

Los electrodos secos montados en un solo módulo (ver Figura 3) y el amplificador de señales SEMG montado sobre este módulo, conforman el sensor SEMG de electrodos secos. Este sensor es usado en el método 2, que se detallan en los apartados siguientes. Esta configuración lo hace fácil de instalar en la superficie del cuerpo, porque el diseño modular simplifica la ubicación, el cableado y no requiere de un gel.

\section{Procesamiento de la señal}

El control de la prótesis robótica consiste en el procesamiento de las señales SEMG adquiridas con la ubicación de tres electrodos en el músculo, dos de estos para la detección del impulso eléctrico en el músculo y el tercero de referencia. Luego, esta señal es convertida dentro del sensor y en el microcontrolador a través de una serie de etapas, las cuales, se ilustran en la Figura 4.

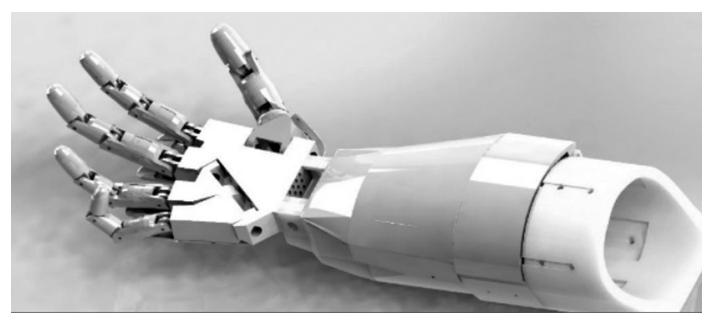

Figura 1. Renderizado de prótesis en software SolidWorks.

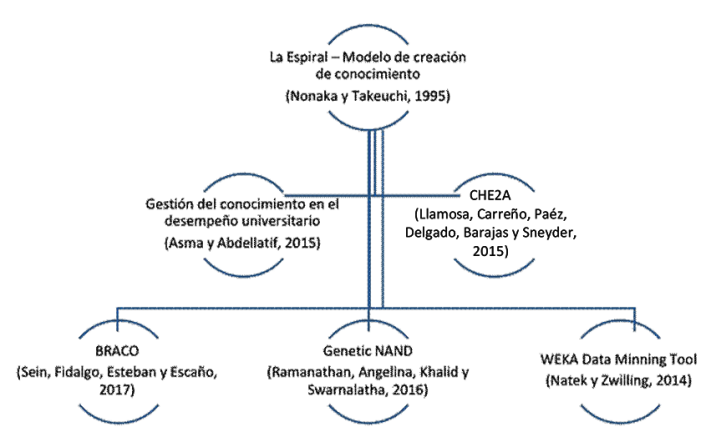

Figura 2. Prótesis impresa con pruebas en su funcionamiento.

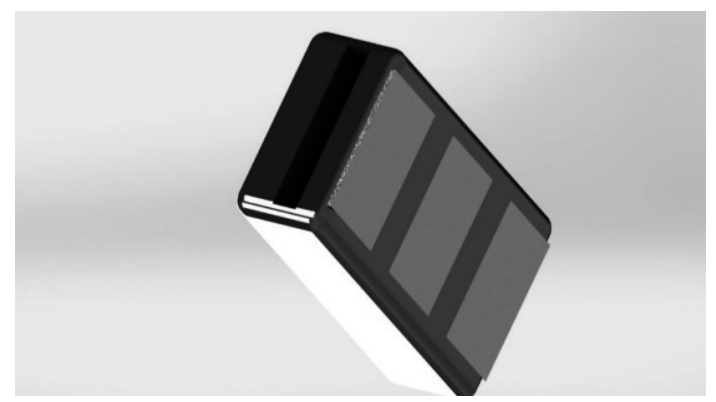

Figura 3. Renderizado del sensor diseñado en el software SolidWorks.

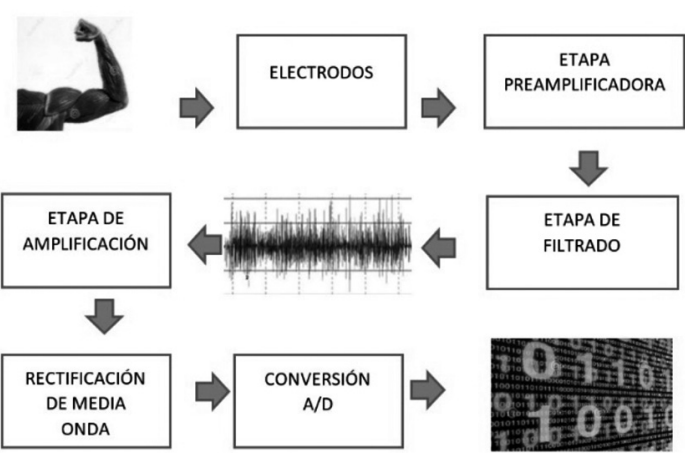

Figura 4. Procesamiento de las señales sEMG. 
Para analizar la variación con respecto al estado del musculo, sea en reposo o cuando éste se contraiga, se utilizó la herramienta serial plotter de Arduino. El impulso eléctrico del músculo es detectado por el amplificador instrumental cuya ganancia puede variar dependiendo de la sensibilidad que se requiera. Las siguientes etapas del sensor se encargan de filtrar la señal, dado que esta señal es contaminada por fuentes de ruido que provienen de la fuente de alimentación, señales de los servomotores o interferencias electromagnéticas. El sensor de señales SEMG es conectado a la tarjeta de control que ejecuta el programa, tanto las lecturas del sensor, aplicación del filtro pasa bajo y el control de los servos se realizaron en el IDE de Arduino. El filtro pasa bajo utilizado es:

$$
\begin{gathered}
H(s)=\frac{w_{c}}{s+w_{c}} \\
Y_{(n)}=\frac{1}{1+w_{c} T} y(n-1)+\frac{w_{c} T}{1+w_{c} T} u(n)
\end{gathered}
$$

donde: " $\mathrm{w}_{\mathrm{c}}$ " es la frecuencia de corte en $\mathrm{rad} / \mathrm{s}$, T es el periodo de muestreo en segundos ( $\mathrm{T}=0,01 \mathrm{~s}$ ), " $\mathrm{y}$ " es la señal de salida y "u" es la señal de entrada. La ecuación (1) es el filtro analógico y la ecuación (2) es su versión digital.

Se realizó el cálculo con una frecuencia de corte de $5,57 \mathrm{~Hz}$, cuyo valor se obtuvo de forma experimental analizando las señales SEMG con la herramienta serial plotter de Arduino. Reemplazando, se halló la ecuación " $y(n)=0,7396 * y(n-1)+0,2604 * u(n)$ ". Teniendo la ecuación se implementó el filtro en el programa de control.

\section{MÉTODOS PARA LA ADQUISICIÓN DE SEÑALES SEMG}

Se trabajó con dos métodos de control. Para el primer método de control de la prótesis el objetivo fue tener la mano abierta o cerrada proporcionalmente utilizando tres electrodos húmedos. En el segundo método de control, se cambió la forma de obtener las señales, prescindiendo de los electrodos húmedos y utilizando electrodos secos. Los electrodos secos estaban montados en una base que contenían dos láminas de acero inoxidable de detección de señales y una tercera de referencia. En este método, se tuvo el objetivo de controlar cada dedo secuencialmente según la intensidad muscular.

\section{Primer método}

Se trabajó la acción de los servomotores y las señales SEMG de forma proporcional, es decir para cada valor detectado correspondía una acción. Sin embargo, el ruido eléctrico en el sistema hacía que el amplificador no logre obtener datos correctos, a pesar del filtro implementado en la tarjeta de control. Por lo tanto, las señales PWM enviadas a los servomotores variaban constantemente y esto llevaba a que los dedos de la prótesis no se quedaran en una posición estable.

Para este método, las señales SEMG fueron captadas utilizando electrodos húmedos superficiales, placas metálicas posicionadas sobre un músculo del antebrazo derecho del sujeto (dos electrodos se ubicaron para detección de impulso eléctrico y un electrodo de referencia) (ver Figura 5). Esta señal fue amplificada utilizando la tarjeta "Muscle sensor v3.0".

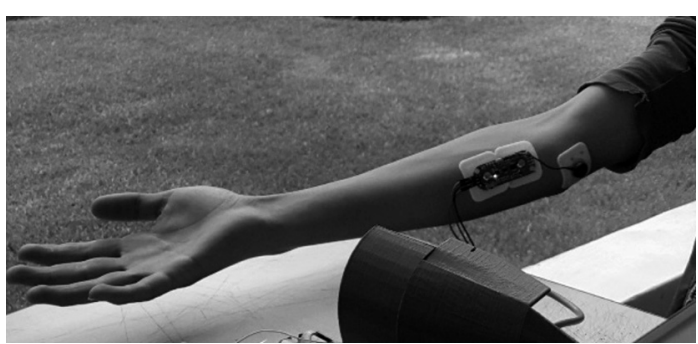

Figura 5. Electrodos superficiales para adquisición de señal del primer método.

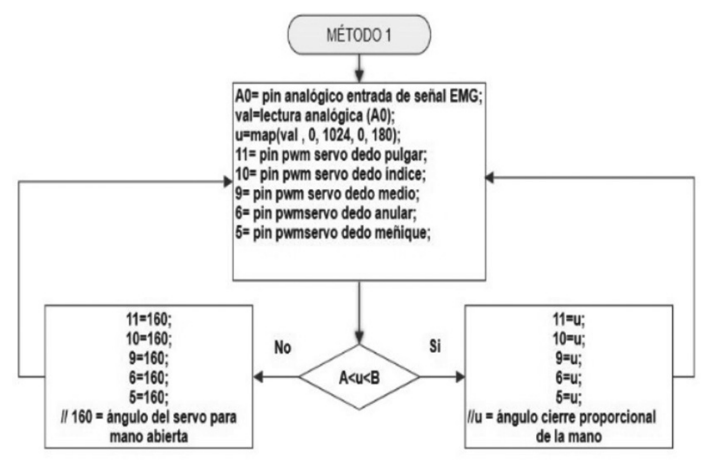

Figura 6. Diagrama de flujo del primer método de control. 
En la Figura 6 se muestra el diagrama de flujo, el cual es la base para el primer método de control. Se declaró los pines de entrada, salida y 2 constantes, de acuerdo con el valor mínimo de la lectura y un valor máximo. El programa se basa en 2 condiciones encargadas de realizar la acción.

\section{Segundo método}

Se utilizó los electrodos secos junto con la tarjeta amplificadora y filtradora "Muscle sensor v3.0" y el filtro pasa bajo ya mencionado. Con esta combinación, se logra una mejora en la calidad de la señal, la cual se muestra más estable y esto da como resultado un movimiento constante. Con una señal más limpia, fue factible un control proporcional de la apertura de la mano robótica en secuencia consecutiva de los dedos de pulgar hacia el meñique. la base del sensor contiene 3 placas de material acero inoxidable (ver Figura 7 y Figura 8). Este modelo no está relleno de pasta conductiva ni sustancias adhesivas. La señal captada resultó ser más limpia.

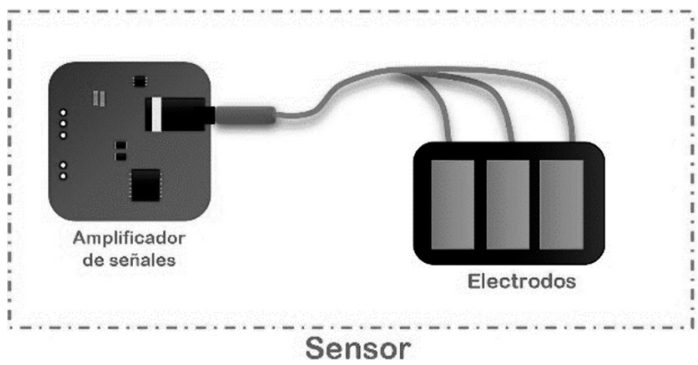

Figura 7. Estructura del sensor de señales SEMG desarrollado para el método 2, el cual incluye al amplificador de señales SEMG y la distribución de los electrodos secos.

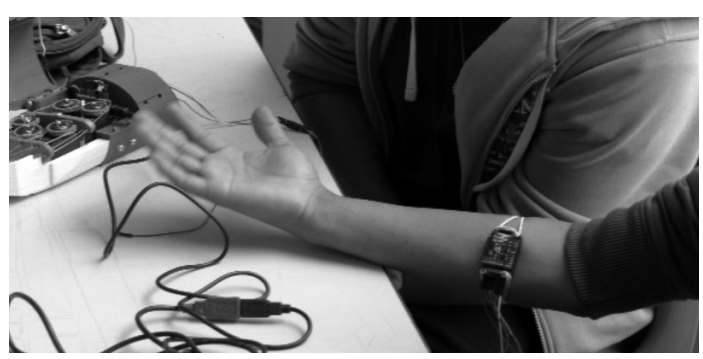

Figura 8. Sensor SEMG desarrollado para el método 2 sobre el musculo del voluntario.
El diagrama de flujo del segundo método se muestra en la Figura 9. Al tenerse una señal más limpia adquirida desde el sensor SEMG de electrodos secos, se obtiene baja variación de la señal adquirida cuando no hay gran variación en contracción muscular del usuario.

La función map() es la encargada del control proporcional, teniendo desde un valor mínimo, intermedios y máximo, además, al ser una matemática entera, los valores fraccionarios no se toman en cuenta, manteniendo la posición de los dedos de la prótesis y evitando movimientos involuntarios.

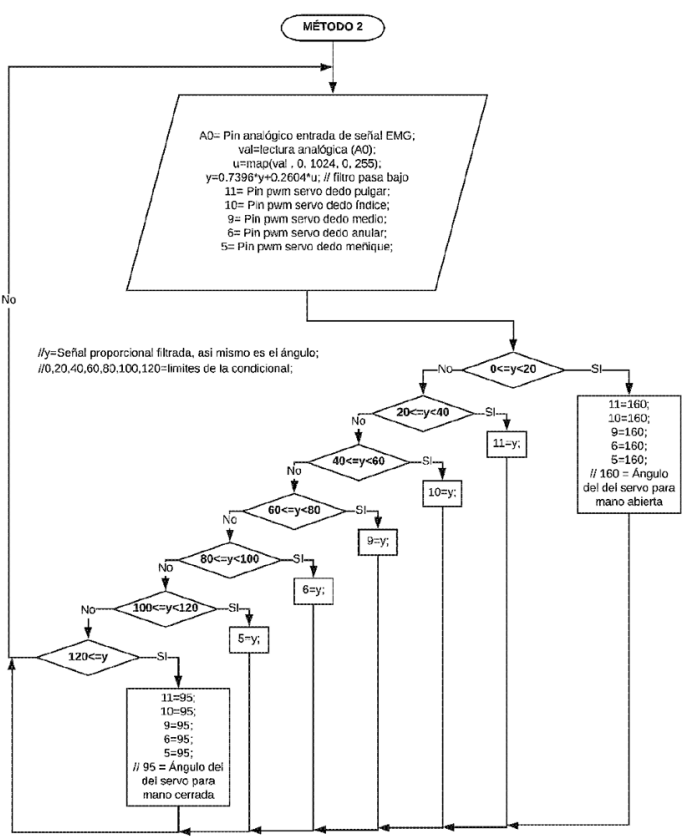

Figura 9. Diagrama de flujo para el segundo método de control.

\section{CONFIGURACIÓN EXPERIMENTAL}

Ambas pruebas se realizaron con 7 voluntarios sin amputación de brazo. En el método 1, el electrodo se coloca en el brazo sobre la zona del músculo palmar mayor [14] (ver Figura 10) y, en el método 2, sobre el musculo flexor común superficial de los dedos [15] (ver Figura 11). Los pasos realizados se detallan a continuación.

Las pruebas del método número 1 consistió, primero, en explicar y demostrar el funcionamiento del sistema sensor y los efectos en la prótesis. Cuando el sujeto de 
prueba comprendió el funcionamiento correcto, se le colocó el sensor en el brazo. Luego, se pidió al sujeto de prueba que extendiera la mano para poder realizar la calibración del sensor, ubicándolo correctamente en el brazo del voluntario. Por último, se pidió al voluntario que realizara la apertura y cierre de la mano para poder comprobar los datos obtenidos por el sensor.

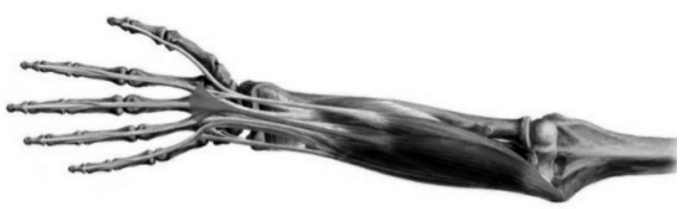

Figura 10. Musculo palmar mayor.

Las pruebas del método número 2, luego de comprobar la correcta lectura de datos del sensor, se solicitó al voluntario que realizara la apertura y cierre de la mano de la prótesis hasta que lograra el control de este movimiento (ver Figura 12). El proceso se repitió para el movimiento de cada dedo, empezando por el pulgar y terminando por el meñique. Se tomó el tiempo de entrenamiento para poder analizar las diferencias entre los electrodos simples (utilizados en el método 1) y los diseñados en este proyecto (utilizados en el método 2).

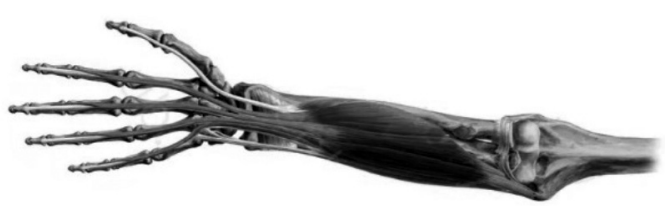

Figura 11. Musculo flexor común superficial de los dedos.

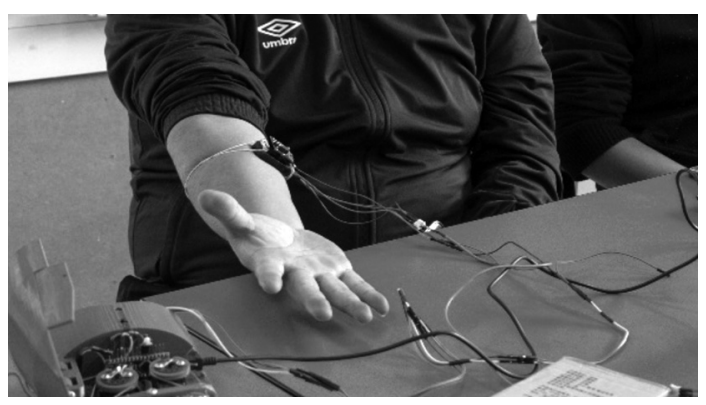

Figura 12. Sensor en la superficie del músculo flexor común superficial de los dedos.

\section{RESULTADOS}

En las Figuras 13-15 se muestran las señales SEMG y el grado de movimiento de los servomotores. El ángulo de los servos varía entre $160^{\circ}$ y $90^{\circ}$, en donde $160^{\circ}$ será la posición que tomará el servo motor para que el dedo se extienda en su totalidad, y $90^{\circ}$ la posición del servomotor para que el dedo se cierre totalmente.

Primer método: Control proporcional de los dedos en conjunto según intensidad muscular En este método los dedos se mueven en conjunto para asegurar el agarre del objeto. En la Figura 13, se muestra la espera de los servomotores y la señal para su calibración en su valor máximo y mínimo. Una vez identificado estos valores, el voluntario regula su intensidad muscular para entender en qué proporción estos se mueven.

\section{Descripción de las señales:}

A. Señal SEMG y servos a la espera de su calibración.

B. Señal calibrada en su valor máximo y mínimo y su posterior aprendizaje.

C. Aplicación de una fuerte señal SEMG para el cierre de los servomotores.

D. Relajación del músculo y el retorno de los servomotores a la posición abierta.

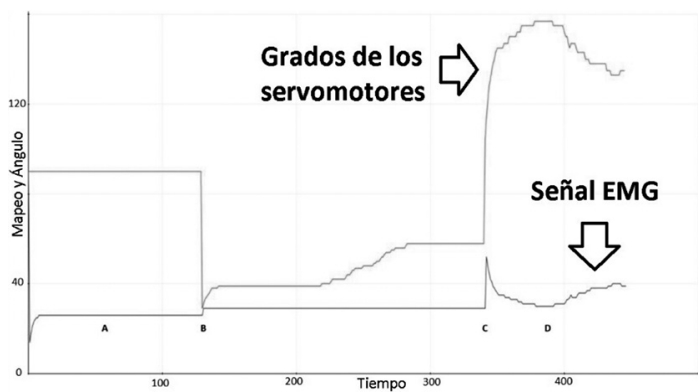

Figura 13. Calibración de señal y aprendizaje de intensidad de la señal.

En la Figura 14, se pueden ver las señales cuando el sujeto de prueba eleva gradualmente la intensidad muscular para observar y aprender en qué medida es posible el control del cierre total de la prótesis. Después de la fase de aprendizaje, el sujeto es capaz de manipular la apertura y cierre de la prótesis a voluntad (ver Figura 15). 


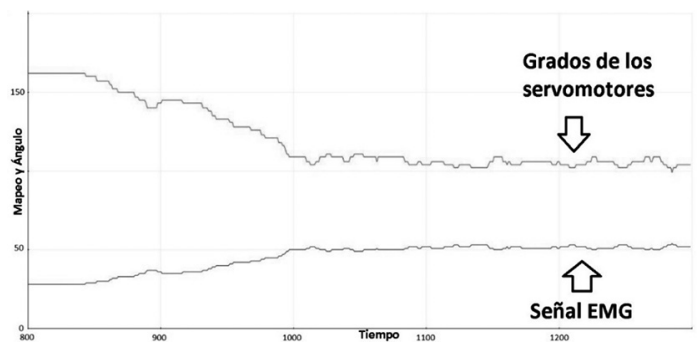

Figura 14. Método 1. Control gradual del cierre de la prótesis.

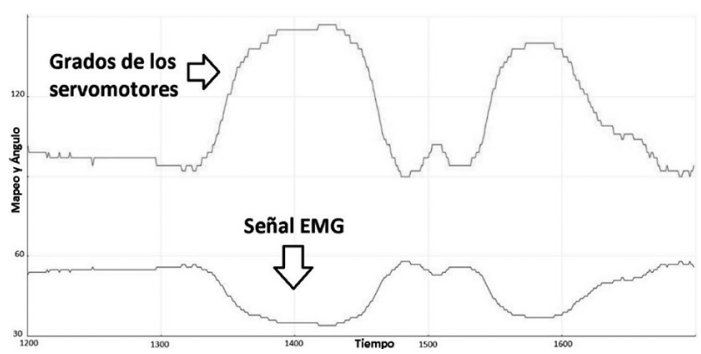

Figura 15. Método 1. Control a voluntad de la apertura y cierre de la prótesis.

Finalmente, se solicitó a los voluntarios lograr el control del cierre en $65 \%$ y $33 \%$, aproximadamente, además, de la apertura y cierre total de la prótesis. Y se registró el tiempo, en minutos, que les tomó aprender a realizar estos movimientos, siendo siempre el músculo de referencia el Palmar Largo. Se obtuvo como resultado del método 1 los datos mostrado en la Tabla 1.

Interpretando los datos de la Tabla 1 en gráficos, tenemos que el aprendizaje mínimo y máximo de la apertura y cierre de la mano fue de 0,50 min y 8 min, respectivamente (ver Figura 16); el aprendizaje del movimiento de cierre al $33 \%$ fue entre $2 \mathrm{~min}$ y 10 min con alta dispersión (ver Figura 17); el aprendizaje del movimiento de cierre al $65 \%$ fue entre 2 min y 10 min (ver Figura 18); el tiempo de aprendizaje de control total de la prótesis fue de 10 min como máximo (ver Figura 19); todos los voluntarios lograron un control de más de $50 \%$ y 4 personas en la prueba a un $100 \%$ (ver Figura 20).

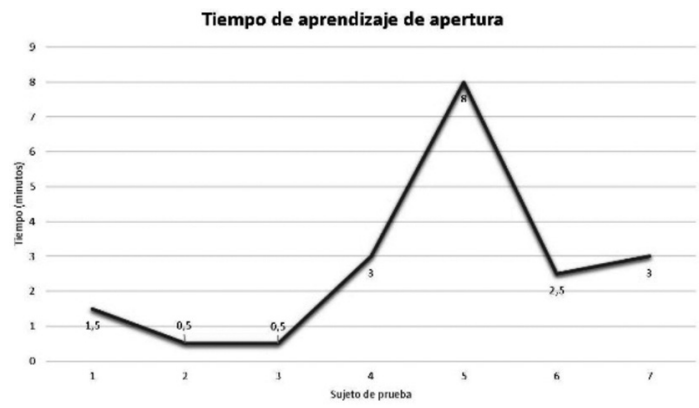

Figura 16. Tiempo de aprendizaje de apertura y cierre. Todos los voluntarios aprendieron a controlar la prótesis. El tiempo máximo registrado para el aprendizaje de la apertura y cierre fue 8 minutos para el método 1 .

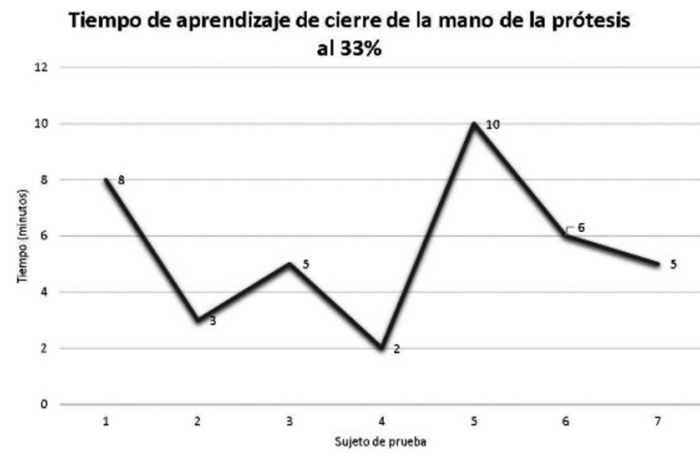

Figura 17. Tiempo de aprendizaje de cierre al $33 \%$. Todos los voluntarios aprendieron a controlar la prótesis. El tiempo máximo de aprendizaje de cierre al 33\% fue 10 minutos.

Tabla 1. Registro de datos de la prueba con el método 1 .

\begin{tabular}{|c|c|c|c|c|c|c|}
\hline Voluntario & $\begin{array}{c}\text { Tiempo de } \\
\text { apertura }\end{array}$ & $\begin{array}{c}\text { Tiempo de } \\
\text { cierre }\end{array}$ & $\begin{array}{c}\text { Tiempo } \\
\mathbf{3 3 \%}\end{array}$ & $\begin{array}{c}\text { Tiempo } \\
\mathbf{6 5 \%}\end{array}$ & $\begin{array}{c}\text { Tiempo } \\
\text { Aprendizaje total }\end{array}$ & $\begin{array}{c}\text { Grado de } \\
\text { manejo alcanzado }\end{array}$ \\
\hline 1 & 1,50 & 1,50 & 8,00 & 6,00 & 10 & $100 \%$ \\
\hline 2 & 0,50 & 0,50 & 3,00 & 6,50 & 8 & $100 \%$ \\
\hline 3 & 0,50 & 1,00 & 5,00 & 6,50 & 10 & $100 \%$ \\
\hline 4 & 3,00 & 3,00 & 2,00 & 2,00 & 5 & $100 \%$ \\
\hline 5 & 8,00 & 8,00 & 10,00 & 10,00 & 10 & $75 \%$ \\
\hline 6 & 2,50 & 2,50 & 6,00 & 7,00 & 7 & $75 \%$ \\
\hline 7 & 3,00 & 3,00 & 5,00 & 7,00 & 10 & $50 \%$ \\
\hline
\end{tabular}




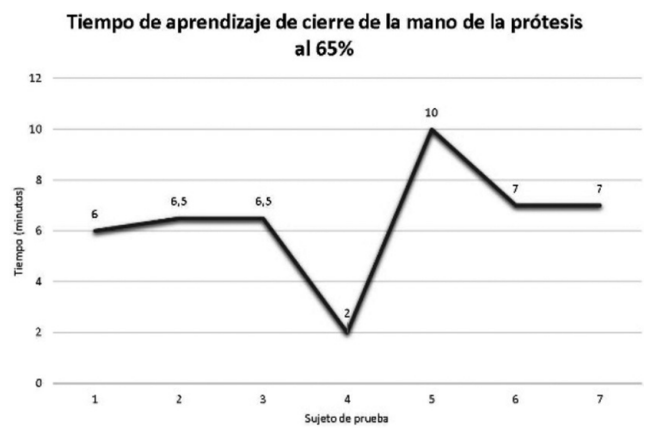

Figura 18. Tiempo de aprendizaje de cierre al $65 \%$. Todos los voluntarios aprendieron a controlar la prótesis. El tiempo máximo de aprendizaje de cierre al $65 \%$ fue 10 minutos.

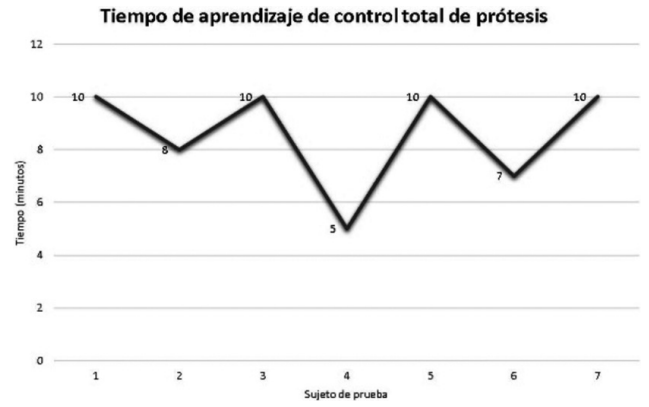

Figura 19. Tiempo de aprendizaje del control total de la prótesis. Todos los voluntarios aprendieron a controlar la prótesis. El tiempo máximo de aprendizaje registrado fue $10 \mathrm{~min}$.

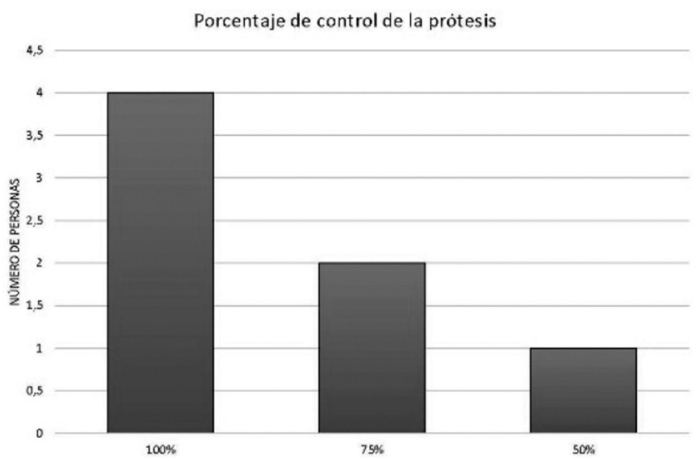

Figura 20. Porcentaje de control de la prótesis. Todos los voluntarios aprendieron a controlar la prótesis. 4 lograron un control al 100\%, 2 a un $75 \%$ y 1 al $50 \%$.

Segundo método: Control para cada dedo en rangos específicos según intensidad muscular

En este segundo método los servomotores accionaron la apertura y cierre de los dedos en forma gradual.
A medida que la señal SEMG aumenta, el cierre se ejecuta proporcionalmente, comenzando con el dedo pulgar hacia el dedo índice, y en ese orden hasta que la señal alcance su máximo valor. La apertura de la prótesis es en orden inverso, del dedo meñique hacia el pulgar a medida que la señal baje al mínimo valor. En la Figura 21 se muestran estos movimientos, siendo:
A. Dedo pulgar cerrándose.
B. Dedo índice cerrándose.
C. Dedo medio cerrándose.
D. Dedo anular cerrándose.
E. Dedo meñique cerrándos.e
F. Descenso de la intensidad de la señal SEMG y retorno de los dedos a su posición de reposo - dedos abiertos.

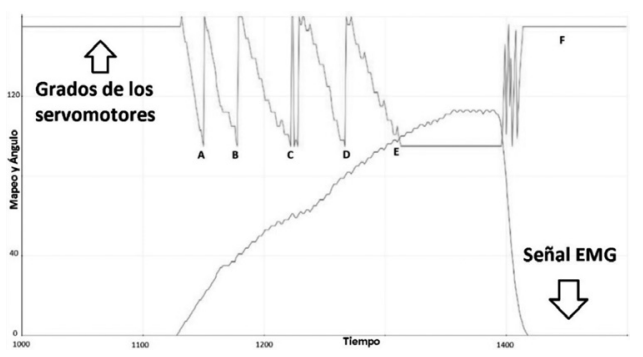

Figura 21. Intensificación de señal SEMG y cierre gradual de los dedos de la prótesis.

En la Tabla 2 se muestra el registro de datos de las pruebas con el método 2 . Interpretando los datos de la Tabla 2 en gráficos, tenemos que el aprendizaje de la apertura y cierre de la mano fue entre $0,43 \mathrm{~min}$ y 3,43 min, con gran dispersión (ver Figura 22). El aprendizaje del movimiento del dedo pulgar fue entre 1,72 min y 2,8 min con baja dispersión (ver Figura 23). En cuanto al aprendizaje del dedo índice, el tiempo de aprendizaje fue entre 0,2 min y 6,4 $\min$ (ver Figura 24), donde el voluntario número 7 tiene dificultad en obtener este movimiento, mientras que los demás aprendieron muy rápido. El aprendizaje para mover el dedo medio también fue rápido, en menos de 1,17 min, mientras que el voluntario 7 no consiguió mover este dedo por lo que en el gráfico se muestra con 0,0 (ver Figura 25). Resultados similares se obtuvieron en el aprendizaje de los dedos anular y meñique, como se muestran en las Figuras 26 y 27. En la Figura 28, se muestra el tiempo total de aprendizaje del control de la prótesis, y en la Figura 29, se ilustra el porcentaje de personas que manejó la prótesis al $100 \%$. 
Tabla 2. Registro de datos de las pruebas con el método 2.

\begin{tabular}{|c|c|c|c|c|c|c|c|c|}
\hline Sujeto & $\begin{array}{c}\text { Tiempo } \\
\text { apertura/ cierre }\end{array}$ & $\begin{array}{c}\text { Tiempo } \\
\text { pulgar }\end{array}$ & $\begin{array}{c}\text { Tiempo } \\
\text { índice }\end{array}$ & $\begin{array}{c}\text { Tiempo } \\
\text { medio }\end{array}$ & $\begin{array}{c}\text { Tiempo } \\
\text { anular }\end{array}$ & $\begin{array}{c}\text { Tiempo } \\
\text { meñique }\end{array}$ & $\begin{array}{c}\text { Tiempo } \\
\text { total }\end{array}$ & $\begin{array}{c}\text { Proporción } \\
\text { manejo }\end{array}$ \\
\hline 1 & 1,70 & 2,08 & 1,28 & 0,28 & 0,35 & 1,92 & 7,62 & $100 \%$ \\
\hline 2 & 0,43 & 1,72 & 1,50 & 1,00 & 0,18 & 0,65 & 5,48 & $100 \%$ \\
\hline 3 & 1,50 & 2,25 & 1,57 & 0,83 & 2,50 & 1,08 & 9,73 & $100 \%$ \\
\hline 4 & 2,40 & 2,80 & 2,50 & 1,03 & 0,67 & 1,58 & 10,99 & $100 \%$ \\
\hline 5 & 0,88 & 2,53 & 1,50 & 1,17 & 0,40 & 0,82 & 7,30 & $100 \%$ \\
\hline 6 & 3,43 & 2,18 & 0,20 & 0,27 & 0,18 & 0,15 & 6,42 & $100 \%$ \\
\hline 7 & 2,58 & 1,60 & 6,40 & - & - & - & 10,58 & $50 \%$ \\
\hline
\end{tabular}

En las pruebas se observó que para el mejor aprendizaje del control se debe aprender a controlar el cierre y apertura total de la prótesis para luego controlar los dedos.

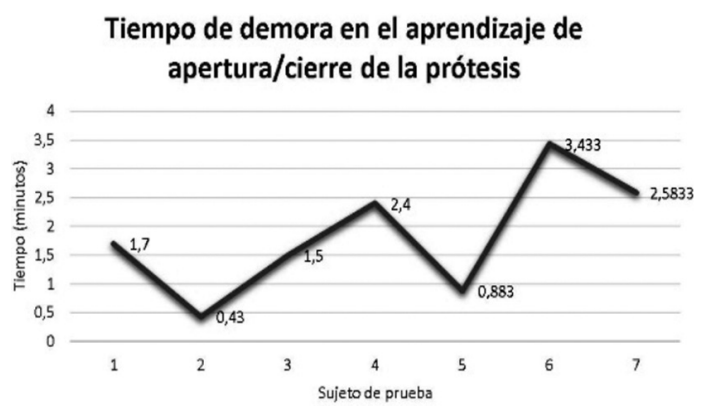

Figura 22. Tiempo de demora en el aprendizaje de apertura/cierre de la prótesis. Todos los voluntarios aprendieron a controlar la prótesis. El tiempo máximo registrado para el aprendizaje de la apertura y cierre fue de 3,43 minutos para el método 2 .

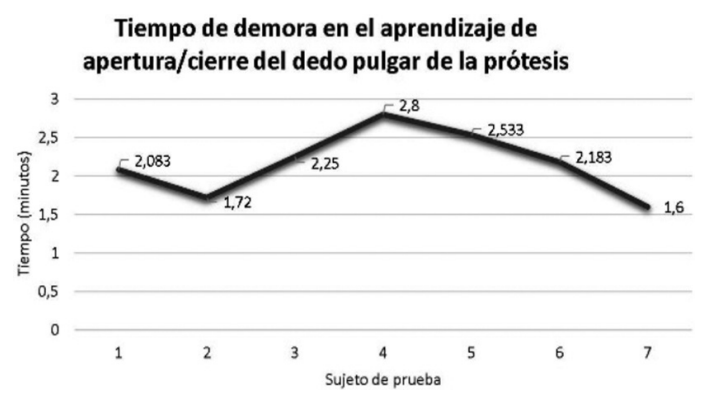

Figura 23. Tiempo de demora en el aprendizaje de apertura/cierre del dedo pulgar de la prótesis. Todos los voluntarios aprendieron a controlar la prótesis. El tiempo máximo registrado para el aprendizaje de la apertura y cierre del dedo pulgar fue de 2.80 minutos.
Tiempo de demora en el aprendizaje de apertura/cierre del dedo índice de la prótesis

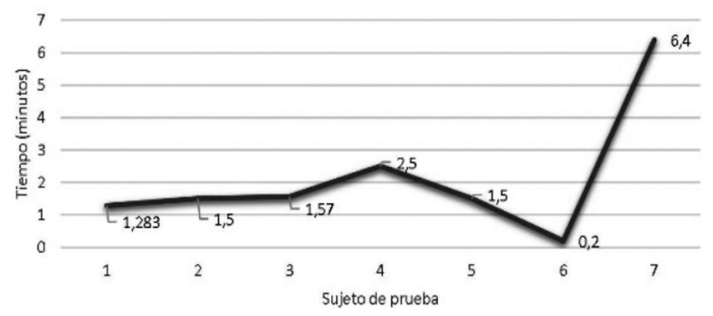

Figura 24.Tiempo de demora en el aprendizaje de apertura/cierre del dedo índice de la prótesis. Todos los voluntarios aprendieron a controlar la prótesis. El tiempo máximo registrado para el aprendizaje de la apertura y cierre del dedo índice fue de 6,40 minutos.

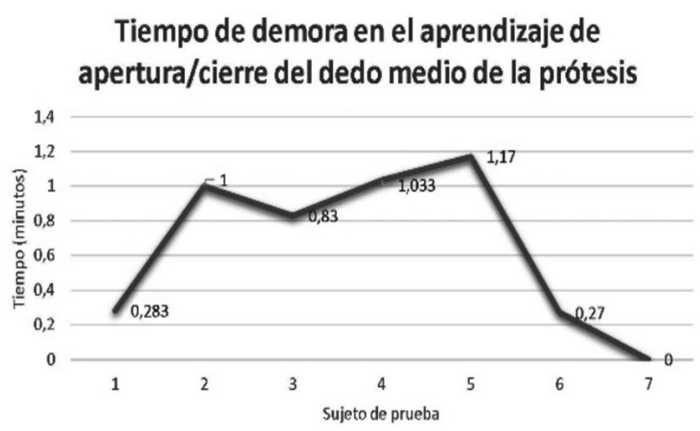

Figura 25.Tiempo de demora en el aprendizaje de apertura/cierre del dedo medio de la prótesis. Seis de los siete voluntarios lograron el aprendizaje, y el tiempo máximo registrado para el aprendizaje de la apertura y cierre del dedo medio fue de 1,17 minutos. 


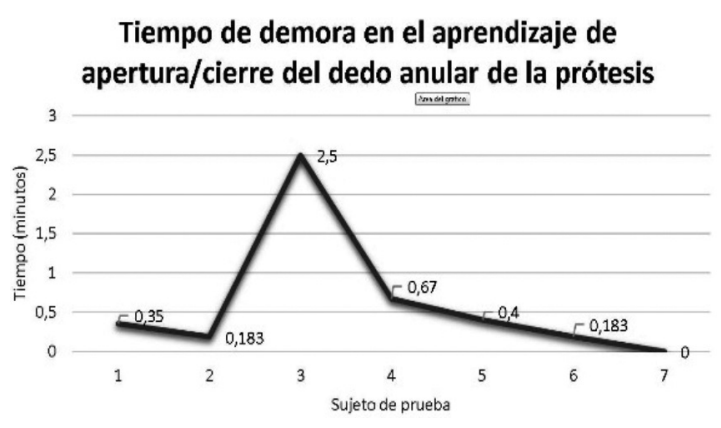

Figura 26. Tiempo de demora en el aprendizaje de apertura/cierre del dedo anular de la prótesis. Seis de los siete voluntarios lograron el aprendizaje, y el tiempo máximo registrado para el aprendizaje de la apertura y cierre del dedo anular fue de 2,50 minutos.

Tiempo de demora en el aprendizaje de apertura/cierre del dedo meñique de la prótesis

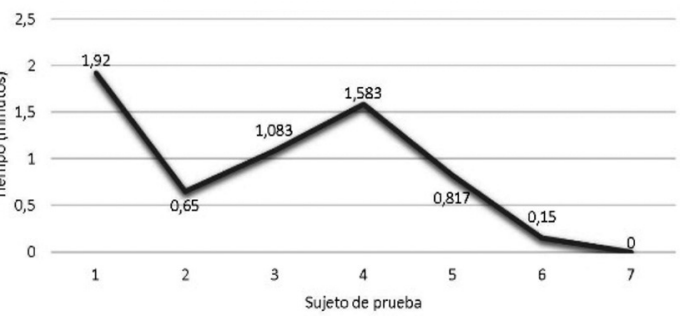

Figura 27. Tiempo de demora en el aprendizaje de apertura/cierre del dedo meñique de la prótesis. Seis de los siete voluntarios lograron el aprendizaje, y el tiempo máximo registrado para el aprendizaje de la apertura y cierre del dedo meñique fue de 1,92 minutos.

Tiempo de demora en el aprendizaje de control total de la prótesis

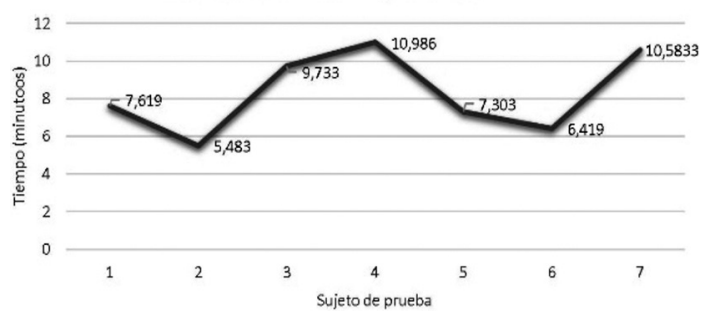

Figura 28. Tiempo de demora en el aprendizaje de control total de la prótesis. Seis de los siete voluntarios lograron el aprendizaje total de la prótesis y el tiempo máximo registrado fue de 10,99 minutos, mientras el sujeto número siete logró un control parcial de la prótesis en un tiempo de 10,58 minutos.

\section{Control de la prótesis}

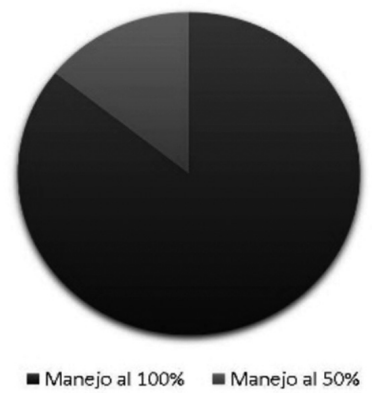

Figura 29. Porcentaje del control total de la prótesis. Seis de los siete voluntarios lograron un manejo al $100 \%$ de la prótesis.

En la Figura 30 se muestra una imagen del entrenamiento del voluntario, donde se puede observar que el voluntario alcanzó a manejar la prótesis al 100 por ciento. La prótesis responde bien al control de los voluntarios; en algunos casos la prótesis sigue muy de cerca el movimiento de los dedos de los voluntarios.

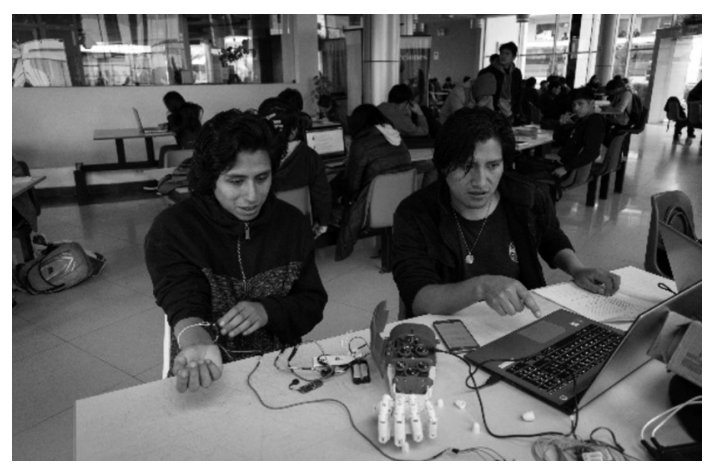

Figura 30. Voluntario de prueba 6 del método 2 obteniendo el control al $100 \%$ de la prótesis.

Resultado de la evaluación de la comodidad de uso del electrodo en el método 1 - electrodos superficiales tipo $\mathbf{A g} / \mathbf{A g C l}$

En la Figura 31, se muestra el número de veces que se calibró la ganancia del sensor para obtener el rango adecuado en el control de la prótesis. Dado que los electrodos tienen pegamento y pasta conductora, no es posible deslizar estos en relación con el musculo, lo que dificulta su ubicación.

En la Figura 32, se puede observar que después de las pruebas realizadas, las personas manifiestan que 
es poco cómodo el manejo de la prótesis utilizando los electrodos húmedos.

Resultado de la evaluación de la comodidad de uso del electrodo en el método 2 - Electrodo seco En la Figura 33, se muestra el número de calibraciones que se realizó modificando la posición del electrodo seco en relación con el musculo sin modificar la ganancia del sensor.

En la Figura 34, se puede observar que después de la prueba con los voluntarios, ellos manifiestan su comodidad en el manejo de la prótesis utilizando los electrodos secos diseñados en la presente investigación.

\section{Cambio del control en el tiempo de la prótesis con electrodos secos}

Es bien sabido que los electrodos secos tienen la desventaja de cambiar su impedancia a medida que pasa el tiempo [9, 11]. Uno de los más detallados estudios acerca de los diferentes tipos de adquisición de señales SEMG lo realizaron A. Searle y L. Kirkup [16] donde compararon 4 tipos de electrodos, tres electrodos secos de diferentes metales que fueron de aluminio, acero inoxidable y titanio, y los electrodos húmedos de $\mathrm{Ag} / \mathrm{AgCl}$. Registraron el efecto de la impedancia en función al tiempo que se muestra en la Figura 35. En este resultado del experimento, se observa que el electrodo húmedo mantiene su impedancia con respecto al tiempo y los electrodos secos de titanio, aluminio y acero inoxidable tienden a disminuir.

Es importante mencionar el efecto de la impedancia de contacto de los diferentes electrodos se traduce como el control para las diferentes aplicaciones en prótesis mioeléctricas, si la impedancia es variable el control de la prótesis podría no ser efectiva, pero si la impedancia es constante la prótesis podrá ser manipulada acorde las necesidades del voluntario. El estudio de Searle y L. Kirkup [16] determina que los electrodos húmedos de $\mathrm{Ag} / \mathrm{AgCl}$ han demostrado mantener el requerimiento de la impedancia constante en función al tiempo el cual favorece el control de Prótesis.

Sin embargo, en las pruebas realizadas en la presente investigación, esta impedancia no afecta el control de la prótesis, por el contrario, mejora su control al tener menos impedancia a medida que pasa el

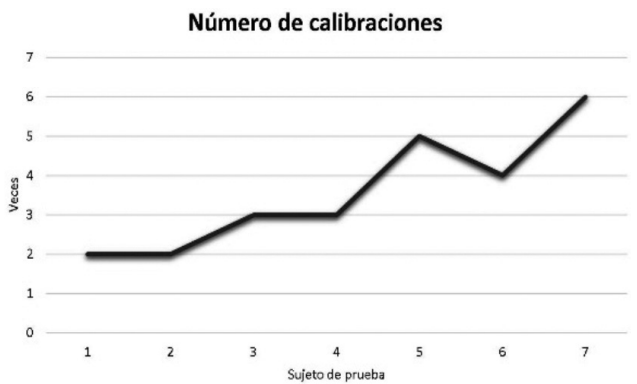

Figura 31. Número de calibraciones realizadas para obtener una señal sEMG manipulable en el método 1.

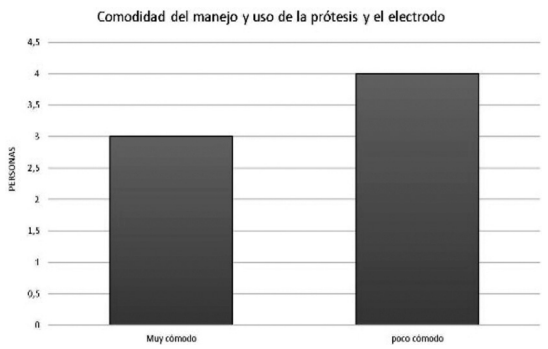

Figura 32. Comodidad de manejo de la prótesis con el electrodo superficial $\mathrm{Ag} / \mathrm{AgCl}$.

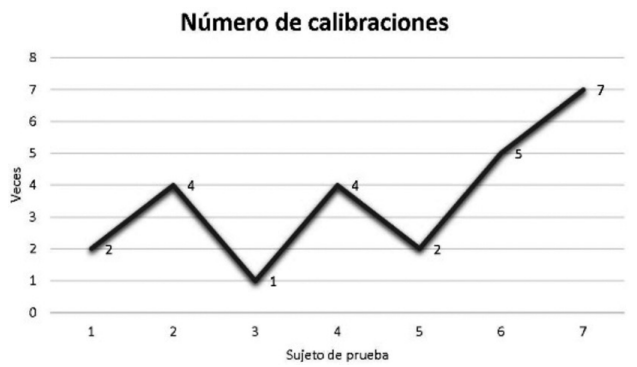

Figura 33. Número de calibraciones realizadas para obtener una señal sEMG manipulable en el método 2 .

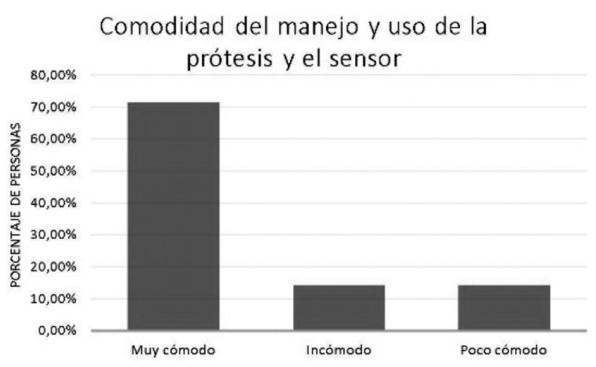

Figura 34. Comodidad del manejo de la prótesis con el sensor de electrodos secos diseñado e implementado para el proyecto. 
Fuente de imagen ftp://ftp.uady.mx/pub/tmp/ smagana/TESIS\%20FINALIZADA.pdf

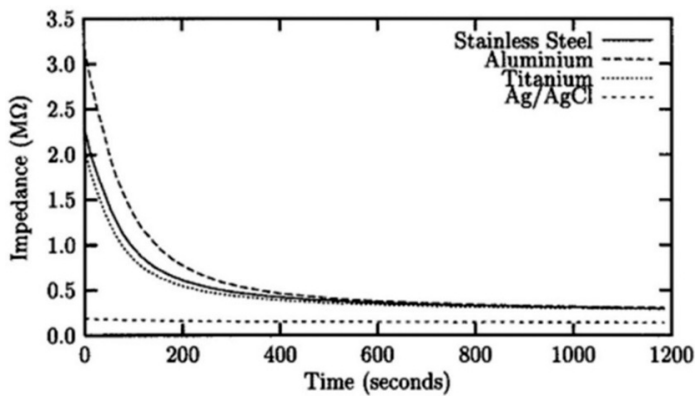

Figura 35. Resultado de la impedancia de contacto entre la piel y el electrodo contra el tiempo para cuatro tipos de electrodos.

tiempo. La razón es que el ser humano se adapta en el control de la prótesis utilizando para ellos la realimentación visual del movimiento de la prótesis. En las Figuras 36-39, se puede observar que los voluntarios controlan sin problemas la prótesis a pesar del paso del tiempo. A partir de 15 min la impedancia de un electrodo seco se mantiene prácticamente constante [11], por lo tanto, se espera que a partir de 15 min el control de la prótesis no varíe.

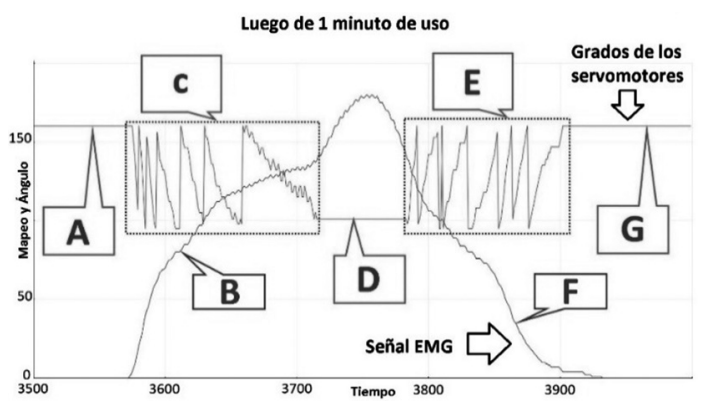

Figura 36. Resultados de las pruebas del control de la prótesis utilizando los electrodos secos, luego de pasado 1 min de instalados los electrodos en el voluntario.

En la Figura 36:

A. Servos en $160^{\circ}$ (mano abierta).

B. Contracción muscular.

C. Cierre proporcional de cada dedo de la prótesis.

D. Servos en $95^{\circ}$ (mano cerrada).

E. Apertura proporcional de cada dedo de la prótesis.

F. Expansión muscular.

G. Servos en $160^{\circ}$ (mano abierta).

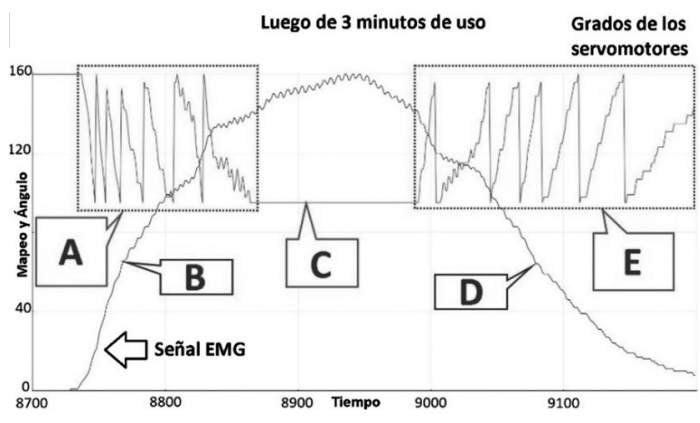

Figura 37. Resultados de las pruebas del control de la prótesis utilizando los electrodos secos, luego de pasado 3 min de instalado los electrodos en el voluntario.

En la Figura 37:

A. Cierre proporcional de cada dedo de la prótesis.

B. Contracción muscular.

C. Servos en $95^{\circ}$ (mano cerrada).

D. Expansión muscular.

E. Apertura proporcional de cada dedo de la prótesis.

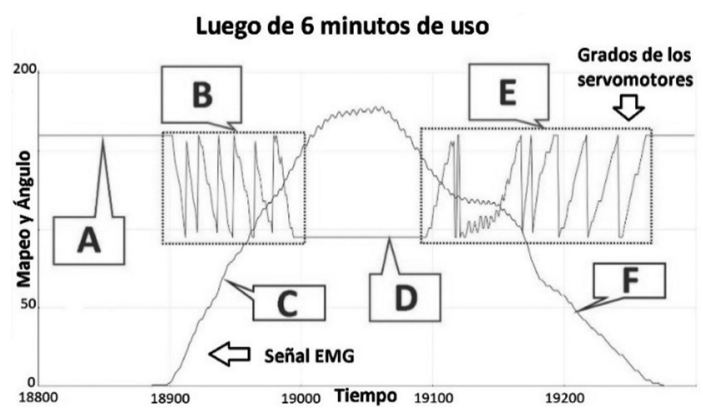

Figura 38. Resultados de las pruebas del control de la prótesis utilizando los electrodos secos, luego de pasado 6 min de instalado los electrodos en el voluntario.

En la Figura 38:

A. Servos en $160^{\circ}$ (mano abierta).

B. Cierre proporcional de cada dedo de la prótesis.

C Contracción muscular.

D. Servos en $95^{\circ}$ (mano cerrada).

E. Apertura proporcional de cada dedo de la prótesis.

F. Expansión muscular.

En la Figura 39:

A. Servos en $160^{\circ}$ (mano abierta).

B. Cierre proporcional de cada dedo de la prótesis. 


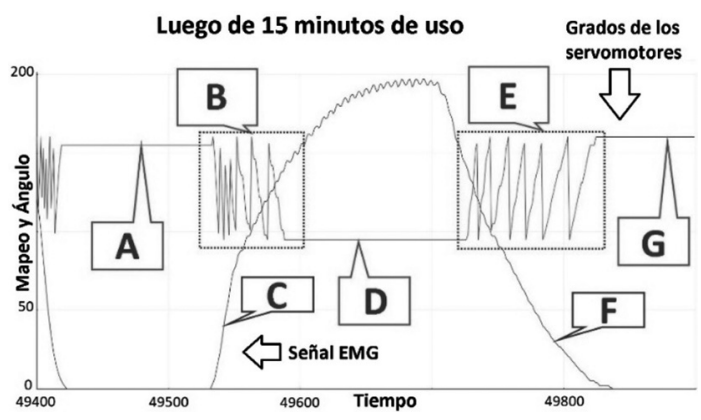

Figura 39. Resultados de las pruebas del control de la prótesis utilizando los electrodos secos, luego de pasado 15 min de instalado los electrodos en el voluntario.

C. Contracción muscular.

D. Servos en $95^{\circ}$ (mano cerrada).

E. Apertura proporcional de cada dedo de la prótesis.

F. Expansión muscular.

G. Servos en $160^{\circ}$ (mano abierta).

\section{DISCUSIÓN DE RESULTADOS}

El trabajo de A. Searle y L. Kirkup [16] compara electrodos secos y húmedos, sin embargo, no realiza un estudio del uso de estos electrodos en una prótesis real. Hakonen [17] realiza la comparación de electrodos secos y húmedos, llegando a la conclusión que los electrodos secos de $\mathrm{Ag} / \mathrm{AgCl}$ sintetizado son comúnmente utilizados por su alta estabilidad, sin embargo, no realiza un análisis sobre un electrodo seco de acero inoxidable. A diferencia de los trabajos previos, en el presente trabajo se demuestra que un electrodo seco de la configuración de acero inoxidable permite el control de una prótesis, porque la variación de la impedancia (común en este tipo de electrodos) no afecta el control, dado que las personas se adaptan a estos cambios. La ventaja de utilizar un electrodo de acero inoxidable es su fabricación simple en comparación de los electrodos secos de $\mathrm{Ag} / \mathrm{AgCl}$ sintetizado. Además, es posible mover los dedos en forma gradual y secuencial con un solo sensor de electrodos secos. Si bien, no se mueven los dedos en forma independiente, se demuestra que el voluntario puede controlar sin problemas el grado de apertura y cierre de la mano.

En la Tabla 3, se muestran 7 prótesis destacadas comerciales. De las prótesis que poseen la más alta tecnología, tres de ellas utilizan un arreglo de seis electrodos secos (tres para cada musculo), como se muestra en la Figura 40. Mientras que, las prótesis fabricadas en impresoras 3D y con tecnología asequible, para la mayoría de personas, utilizan los electrodos húmedos como medio de adquisición de señales. Los electrodos húmedos requieren, para su funcionalidad, que sean reemplazados cada día, debido a que son de un solo uso. Además, diariamente la persona tendrá que ubicar los electrodos en la distribución correcta para que éste tenga la impedancia adecuada, y en caso falle, quitarse el electrodo, lo que no resulta agradable porque éste posee un pegamento. Por último, posicionar la prótesis, tomando un tiempo considerable para que pueda comenzar su labor. En el sistema de los electrodos secos el propietario de una prótesis solo toma la cinta que contenga los electrodos o la prótesis completa con los electrodos en su interior, se lo coloca en un músculo ya determinado, realiza las pruebas de funcionamiento de la prótesis y comienza sus labores. Un sistema similar se propone en la presente investigación, sin embargo, con un sensor que utiliza tres electrodos secos en un solo módulo y con una fabricación más simple en comparación a las otras similares comerciales.

Tabla 3. Prótesis actuales en el mercado.

\begin{tabular}{|c|l|l|l|c|}
\cline { 2 - 5 } \multicolumn{1}{c|}{} & Empresa & \multicolumn{1}{c|}{$\begin{array}{c}\text { Modelo de } \\
\text { prótesis }\end{array}$} & $\begin{array}{c}\text { Señal de entrada } \\
\text { generada por }\end{array}$ & $\begin{array}{c}\text { Precio aproximado } \\
\text { en dólares }\end{array}$ \\
\hline 1 & Ottobook & Michelangelo & Electrodos secos & 105.000 \\
\hline 2 & Touch bionics & I-Limb & Electrodos secos & 65.000 \\
\hline 3 & Ottobook & Bebionic & Electrodos secos & 10.500 \\
\hline 4 & - & Cyberhand & Cirugía invasiva & - \\
\hline 5 & Open bionics & Deus Ex & Electrodos húmedos & 3.500 \\
\hline 6 & Open bionics & Dextrus & Electrodos húmedos & 1.200 \\
\hline 7 & Exiii & Hackberry & Sensor ultrasónico y electrodos húmedos & 1.200 \\
\hline
\end{tabular}

Fuente de la imagen: https://www.youtube.com/watch?v=q7Am1w-MqRI 


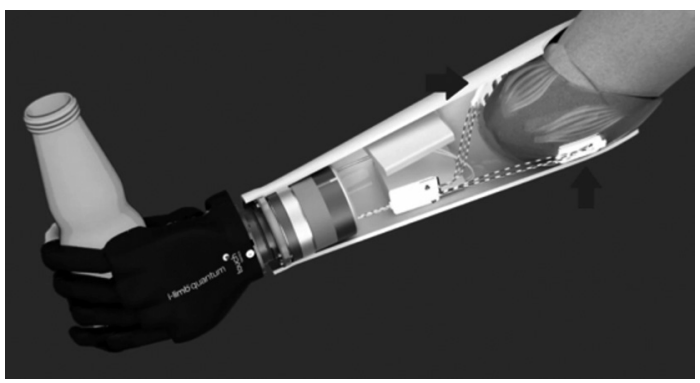

Figura 40. Electrodos secos ubicados en la prótesis I-Limb.

Por último, las prótesis mioeléctricas de bajo costo poseen configuraciones de apertura y cierre totales y botones para sus diferentes configuraciones. Mediante el sistema de adquisición de señales SEMG de la presente investigación, mejoramos el control según la intensidad muscular los dedos para la apertura y cierre gradual de la mano, todo esto con un tiempo mínimo y sin mayor esfuerzo para la mayoría de los voluntarios.

\section{CONCLUSIONES}

El cambio de impedancia, propio de electrodos secos, no afectó el control de la prótesis, por el contrario, se ve una mejora del control, dado que la impedancia tiende a disminuir a medida que pasa el tiempo y la persona se adapta automáticamente a estos nuevos niveles de señales mioeléctricas medidas. Demostrándose que el método 2 (con electrodos secos) es el más efectivo, porque logro un $85,71 \%$ de control total con respecto al $57,14 \%$ del método 1 (con electrodos húmedos), también, porque por medio del sistema de cierre gradual de cada dedo, de pulgar hacia el meñique, las personas pueden adaptarse mejor a este control.

El sensor que contiene los electrodos secos, desarrollado en la presente investigación, y la prótesis para personas con nivel de amputación del tercio proximal en el brazo, desarrollada a partir de la mano del robot InMoov, no requieren tener un proceso complicado de fabricación como las prótesis tradicionales en el mercado, dado que pueden replicarse con materiales comunes y una impresora 3D.

En investigaciones futuras, se pretende implementar nuevos diseños de prótesis que en su conjunto tengan los electrodos secos internamente y mantenga la ventaja actual de ser de bajo costo y de uso viable, además de utilizar otros algoritmos para lograr interpretar de manera más adecuada las señales SEMG de cada dedo de la mano y mover una prótesis artificial con absoluta voluntad.

\section{AGRADECIMIENTOS}

Agradecemos a la Universidad Continental por financiar el desarrollo de la presente investigación.

\section{REFERENCIAS}

[1] Shyamala B. "Real time Electronic Control of Multi Fingered Hand Based on Sensors". Procedia Computer Science. 2016. Vol. 92, pp. 253-259. 2016.

[2] Abu Kasim M.A., Low CH.Y., Ayub M.A., Che Zakaria N.A., Mohd Salleh M.H., Johar, K. and Hamli H. "User Friendly LabVIEW GUI for Prosthetic Hand Control using Emotiv EEG Headset". Procedia Computer Science. Vol. 105, pp. 276-281. 2017.

[3] Quinayás Burgos C.A. y Gaviria-López C.A. "Sistema de identificación de intención de movimiento para el control mioléctrico de una prótesis de mano robótica". Ing. Univ. Vol. 19, pp. 27-50. 2015.

[4] Polishchuk A., Taube Navaraj W., Heidari H. and Dahiya R. "Multisensory Smart Glove for Tactile Feedback in Prosthetic Hand". Procedia Engineering. 2016. Vol. 168, pp. 1605-1608. 2016.

[5] Naim Sidek S., Anija Jalaludin N. and Ubaidah Shamsudin A. "Surface Electromyography (SEMG)-based Thumb-tip Angle and Force Estimation Using Artificial Neural Network for Prosthetic Thumb". Procedia Engineering. Vol. 41, pp. 650-656. 2012

[6] Fazle Rabbi M., Uddin Ahamed N., Alqahtani M. Altwijri O., Hawari Ghazali K. and Sundaraj K. "Investigation of the EMG-angle relationship in the upper-limb muscles during isometric contraction". Biomedical Research. Vol. 28 No 6, pp. 2393- 2397. 2017.

[7] Nikkei, diario. "Japoneses crean prótesis robótica de bajo costo". El Comercio. [Online] 2015. Fecha de consulta: 01 de febrero de 2018. URL: https://elcomercio.pe/tecnologia/inventos/ japoneses-crean-protesis-robotica-costo-229116. 
[8] Brazeiro Jorge, Petraccia Sabrina y Valdés Matías. "MACOSEMU Mano controlada por señales musculares". Montevideo: s.n. 2015.

[9] M. Fatoorechi, R. J. Prance, H. Prance, D. Schwartzman, J. Parkinson and A. Seth. "Comparison of Dry and Wet Electrode Systems for Spontaneous and Event Related Electroencephalograms". International Conference on Sensor Device Technologies and Applications. 2014.

[10] Córdova Ricapa F. "Desarrollo de un sistema para la evaluación de la actividad muscular mediante electrodos de superficie". Ingeniería Electrónica, Pontificia Universidad Católica del Perú. 2013. Tesis.

[11] Cifuentes Gonzáles I.A. "Diseño y construcción de un sistema para la detección de señales electromiográficas". Facultad de Matemáticas, Universidad Autónoma de Yucatan. 2010. Tesis.

[12] Langevin Gael. "InMoov OPEN SOURCE 3D PAINTED LIFE-SIZE ROBOT”. [Online]
2012. Fecha de consulta: 05 de agosto de 2016. URL: http://inmoov.fr/.

[13] Universidad Continental. "Fab Lab UC". [Online] 2017. Fecha de consulta: 10 de enero de 2018. URL: https://ucontinental. edu.pe/fablab/.

[14] “Músculo Palmar Mayor". Anatomia Musculos.org. [Online]. Fecha de consulta: 05 de diciembre de 2017. URL: https://www. musculos.org/musculo-palmar-mayor.html.

[15] "Músculo Flexor Comun Superficial de los Dedos". Anatomia Musculos.org. [Online]. Fecha de consulta: 05 de diciembre de 2017. URL: https://www.musculos.org/musculoflexor-comun-superficial-dedos.html.

[16] Searle A. and Kirkup L. "A direct comparison of wet, dry and insulating bioelectric recordings electrodes". Physiological Measurement. Vol. $21 \mathrm{~N}^{\mathrm{o}}$ 2, pp. 271-283. 2000.

[17] M. Hakonen, H. Piitulainen and A. Visala. "Current state of digital signal processing in myoelectric interfaces and related applications". Vol. 18, pp. 334-359. 2015. 